\title{
A Modern Aesthetic Review of Tujia Hand-waving Dance
}

\author{
Lizhong Wen \\ College of Sports Science \\ Jishou University \\ Jishou, 416000 China \\ Wenlizhong1107@163.com
}

\begin{abstract}
The Tujia hand-waving dance was created by the Tujia people in the long period of production and life, and has been handed down and developed from generation to generation. Throughout the rich traditional Tujia culture, the hand-waving dance, as an indispensable part, has distinct national characteristics. This paper studies the characteristics of Tujia hand-waving dance, which can be divided into large and small according to the number of people; group around to form a circle. And there is a variety of hand waving movements with the same side of the hand swing to show the beauty of dance. We make the Tujia hand-waving dance in the modern society on the big stage blooming glory by digging deep into the physical beauty of the hand dancer, solid health, physical strength, sports and the aesthe tic value of sports in harmony and unity.
\end{abstract}

Keywords-Hand waving dance, Sports aesthetics, Survey

\section{INTRODUCTION}

In the creation of the wisdom of the Tujia people, the hand-waving dance uses its own unique expression way to display the national spirit of Tujia people's unremitting selfimprovement and hard struggle through dancing language, which is concentrated in the colorful national culture of Tujia. Hand-waving dance has become an important link for Tujia people to make emotional connection and inner communication, which not only enriches the national culture with Tujia characteristics, but also gradually becomes the spiritual pillar of Tujia people, having unique significance and value to the social and historical change of Tujia family, which carries the national history and culture and people's life and work. After the baptism of history, potential value of hand-waving dance, such as economic and cultural value, sports fitness value and sports aesthetic value in today's society began to shine, and caught attention widely.

\section{Characteristics OF TUJIA HAND-WAVING DANCE}

\section{A. It can be divided into large and small hand-waving dances according to the number of people}

Tujia hand-waving dance is a kind of dance that exists and develops as a kind of sacrificial activity. In the whole sacrificial activities, the hand-waving dance usually takes a complete form. According to the actual number of participants, the hand-waving dance can be roughly divided into two categories. One is the small hand dance, as the name implies, which means a small number of people and is held in a certain village or a certain ethnic group. It imitates the scenes and actions of the Tujia people working in the fields, and its main purpose is to pay tribute to the land gods and the good men. According to the different holding time, it can be divided into the first month hall, the second month hall, the third month hall, the fourth month hall, the fifth month hall and the twelfth month hall.[1]

The other is the large hand-waving dance, usually involving more than 100 people and according to different villages stand in line. In the sacrifice of the Lord god once every three years, this scene was very grand. It is manifested in the large number of participants, the richness of the dance display and the solemn and sacred ceremony. The team leader first lead the team to raise the high flag of dragon and phoenix and then ignite the torches and all the swing players dance to the drums and constantly change the formation and dance movements..

\section{B. Surround in a group and form a circle}

In a collective dance, a dancer constantly guides the movements of the other swaying players according to the variations of the sound of the gongs and drums. They abide by the leader of the dancer hand in hand, round the circle in the graceful and rhythmic process, and change in the dance of the circle. However, the changes of these movements must be highly consistent with those of the leader, which puts forward very high requirements for the participants' dance skills.

The dance form of the circle reflects the Tujia people's spirit of unity, harmony and concerted efforts in the face of difficulties. This is the crystallization of the wisdom of the Tujia people who have constantly conquered nature and engaged in productive labor in the course of historical changes. They live in a common region and should learn the warm environment in which people are united by the people's Congress. In this way, every Tujia people today are reminded of the importance of great national unity, and constantly strengthen their national pride and identity. Tujia people express this kind of thinking through circular hand- 
waving dance. At the same time it reminds generations of Tujia people to cherish the present state of peace.

\section{The movements of the waving dance are diverse.}

Because the source of hand-waving dance creation is based on the situation of Tujia people in labor, this makes the variety of dance movements inevitable. The imitation of daily life is classified according to image movements as follows. First, dance movements of an entertaining nature, such as "one-handed swing", "two-handed dance" and "loop dance". These dance moves are just good connections and grooming. Secondly, according to the changes of the four seasons, there are different forms of field labor in the field. When spring comes, people are mainly engaged in the activities of loosening the soil, sorting the fields and sowing seeds. These movements are often relatively slow, delicate but little changeable. In summer, people are mainly engaged in weeding and insect removal. And in autumn, the season of harvest, dancing varies according to the type of grain, the movement of harvesting, and so on. So, this is where the swing moves vary. At the same time, the joy of the harvest is full of joy, and the action often shows a great deal of pride and pleasure. Once winter comes, as the weather becomes colder, the Tujia people are more active indoors, and their movements are limited to a certain extent. Third, it reflects people's daily life, such as a girl's dressing up, women's needlework performance, children's various games, and so on. Fourth, to imitate poultry, the Tujia people often raise a lot of poultry, and they also show different movements in different living environments, such as looking for things. Pairwise confrontation and so on is very lively to show their style.

\section{Showing the beauty of the dance with the hand swing on the same side}

To swing hands on the same side is to coordinate the han ds and feet and dance at the same time, according to the rhyt hm of "smooth-crutch",

"Bend" and "shake and sink". One of the most obvious $f$ eatures is the shaking and sinking of the knees. The first bea $t \mathrm{t}$ is the movement of the body first, and then the movement of the foot; the second beat is to move the foot first and then move the right hand; the third beat is to repeat the first beat; the final step is that the foot does not move, and the hands o pen on either side and bend your knees and tremble again an d complete the whole action. In the whole swing change, the amplitude of the swing is increasing which makes the handwaving dance softer with a high aesthetic role.

\section{The Aesthetic Value of Sports In Tujia's WAVING DANCE}

\section{A. Human body beauty}

There are many movements of Tujia ' $s$ hand - waving dance which are imitation of labor , so there is a need for a very powerful hands to perform, which requires both hands to show " hard " in labor . In the process of practicing the hand-waving dance, it is very important to grasp the strength of the human body. The hand-waving dance developed from the changes in labor can not only be used as an aerobic exercise for people to exercise their bodies, but also give play to the aesthetic feeling of the dance to constantly shape themselves. In the process of practicing this kind of dance, it takes a long time to use both hands to keep swinging, so that the flexibility of both hands is improved constantly, and the people who appreciate the waving dance also experience the pleasure of movement while witnessing the beauty of the body.

Nowadays, people are constantly pursuing the bodybuilding image brought about by sports, and the Tujia handwaving dance is a good way to bring this kind of sports aesthetic feeling into the dance, and not only can we realize our own perfect form through continuous hand waving practice, but also feel the power of the hand dance to the body to enjoy the aesthetic.

\section{B. The beauty of solid health}

Tujia people have no fear of difficulties and positive spirit, in the hand-waving dance infinite vitality is revealed, constantly activating the power of people's lives. The action of hand-waving dance is at first some basic, monotonous and mechanical movements, and it is easy to bring a certain weary mood to the person .Therefore, when people inherit and develop the waving dance, some changes have been made, and a lot of sports elements with fitness function have been added, so it is very good to change the monotonicity of the pendulum dance.

Along with people's pursuit of loving sports, the sports factors are gradually added in the process of inheriting the hand-waving dance, so that it is convenient for practitioners to enhance their interest in the practice of hand dancing. Moreover, people who learn this dance will not be restricted by the cultural quality, age level and gender difference, so that everyone can truly participate and experience a healthy activity. Therefore, people in the practice of hand dance is also equivalent to a sports exercise, which is also why the present hand-waving dance can achieve the purpose of a strong body and healthy body and mind.

\section{Beauty of physical strength}

The strength of the hand-waving dance comes from the movement of the hands in the process of dancing, which leads to the movement of the muscles, and constantly overcomes the various obstacles of the body, thus showing the aesthetic sense of strength of the body's movement. In the practice of hand-waving dance, although the physical strength of the person is in a state of exhaustion, but its consumption of this amount is not excessive. So combined with aerobic activities, it in relaxed music relaxes the body, and strength also gets a certain degree of exercise. 
For beginners, because the physical resilience is not very good, so the swing is not too large. As the pace of practice accelerates and time goes by, the flexibility of the body becomes better and better. At the same time, it can be well adapted to the dance requirements in the strength of the show of beauty. For example, when the swing is larger, the strength of the arm increases, thus enhancing the aesthetic feeling of the action.

\section{Beauty of sports}

The aesthetic sense of movement of Tujia hand-waving dance is manifested in the coordination of form and movement. As explained earlier, the Tujia large handwaving dance is a imitation of labor. For example, the joy of the people in harvest, the performance in the hand-waving dance is a big swing of the hand, and then the feet should also cooperate well with the movement of the hand, rotating quickly, and the expression on the face will also change. This series of actions must be complete and coordinated in order to achieve. When these actions are repeated repeatedly, they satisfy the exercise of the ligaments, joints and muscles of the human body, which is also an expression of rich the inner world of Tujia people.

For the small hand-waving dance, the movement is very light, elegant and delicate, mainly reflecting the life picture of Tujia people in leisure. Whether large or small, they conclude simple life content, through the language of dance to show people the life style of Tujia, so its connotation is profound, vivid image.

\section{E. The Beauty of Harmony and Unity}

Sports are the unity of physical coordination and inner peace, which can make the great spirit flow smoothly in the mind. Whether it is an elegant pendulum or a happy double pendulum, Tujia hand-waving dance requires all parts of the body, such as nerves, breathing and joints, to be in a state of motion so that to show the freshness, elegance and symmetry of the dance. When every part of the body gets better exercise, the beauty of harmony and unity of sports and hand-waving dance can be displayed to the fullest extent. Just as people watch a magnificent gymnast compete, the athlete's body must display exquisite skills, but the mind must remain absolutely calm, without losing the aesthetic feeling of movement. Based on this request, the pursuit of harmonious and unified aesthetics of Tujia hand-waving dance is to achieve the unity of body and mind, the unity of emotion and will.

\section{CONCLUSION}

As an important form of expression and carrier of Tujia culture, Tujia hand-waving dance plays a very important role in displaying the profound meaning and the spiritual state of Tujia people to the world through dance language and is also a culture with distinct national characteristics. From the characteristics of Tujia Pendulum Dance, this paper probes into the aesthetic value of sports contained in the hands. In the process of changing hand movements, the beauty of movement is constantly revealed to satisfy the physical and mental pleasure of people, and it can achieve the function of exercising the body. In the new era, the Tujia hand-waving dance is no longer a traditional temple activity to worship gods, but gradually realizes the integration of healthy elements in happiness, which plays an important role in promoting people's healthy physique and body and mind. The hand-waving dance has been accepted by the masses because it's easy to leam. In all kinds of hand waving exercises, the concept of artistic conception and health is deeply contained. We should explore the aesthetic value of the hand-waving dance with a more open mind, inherit and develop it in innovation, promote and popularize the sport constantly, and finally make it benefit people's body and life

\section{REFERENCES}

[1] wang jia. Aesthetic culture and interactive development of tujia traditional sports in physical education teaching [J]. Journal of cultural studies, June 2015 :161-163.(In Chinese)

[2] Yang ting. Tujia hand dance: cultural focus and aesthetic expression [J]. Art,2012,06:179-184.(In Chinese)

[3] luo chenghua, liu safety. The historical inheritance of tujia hand-waving dance is coupled with its habitat [J]. Guizhou social science,2016,01:8891.(In Chinese)

[4] Yang min, Chen guangjiu. Study on the cultural connotation and cultural value of tujia hand-waving dance [J]. Journal of wenzhou university (nature science edition),2012, apr. 55:60.(In Chinese)

[5] shi manli. Study on the function and inheritance path of tujia handwaving dance [J]. Guizhou ethnic studies, Feb. 2015 :71-74.(In Chinese) 